\title{
Erratum to: Data blinding for the nEDM experiment at PSI
}

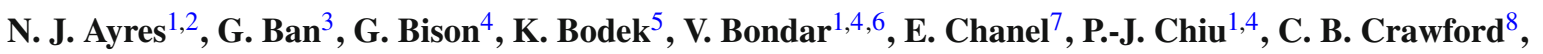 \\ M. Daum ${ }^{4}$, S. Emmenegger ${ }^{1}$, L. Ferraris-Bouchez ${ }^{9}$, P. Flaux ${ }^{3}$, Z. Grujic ${ }^{10,15}$, P. G. Harris ${ }^{2}$, N. Hild ${ }^{1,4}$, J. Hommet ${ }^{3}$,

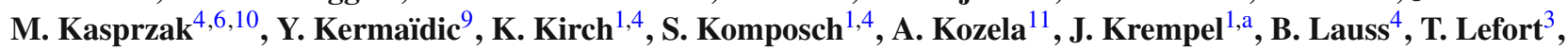 \\ Y. Lemiere ${ }^{3}$, A. Leredde ${ }^{9}$, P. Mohanmurthy ${ }^{1,4}$, A. Mtchedlishvili ${ }^{4}$, O. Naviliat-Cuncic ${ }^{3}$, D. Pais ${ }^{1,4}$, F. M. Piegsa ${ }^{7}$,

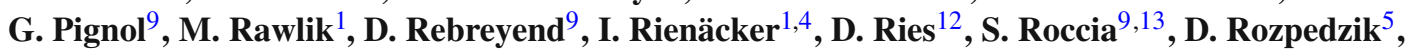 \\ P. Schmidt-Wellenburg ${ }^{4}$, A. Schnabel ${ }^{14}$, R. Virot $^{9}$, A. Weis ${ }^{10}$, E. Wursten ${ }^{6,16}$, J. Zejma ${ }^{5}$, G. Zsigmond ${ }^{4}$ \\ ${ }^{1}$ Institute for Particle Physics and Astrophysics, ETH Zürich, Zürich, Switzerland \\ 2 Department of Physics and Astronomy, University of Sussex, Falmer, Brighton, UK \\ ${ }^{3}$ Normandie Université, ENSICAEN, UNICAEN, CNRS/IN2P3, LPC Caen, Caen, France \\ ${ }^{4}$ Paul Scherrer Institute, Villigen, Switzerland \\ ${ }^{5}$ M. Smoluchowski Institute of Physics, Jagiellonian University in Krakow, Kraków, Poland \\ ${ }^{6}$ Instituut voor Kern- en Stralingsfysica, Katholieke Universiteit Leuven, Leuven, Belgium \\ ${ }^{7}$ University of Bern, Albert Einstein Center for Fundamental Physics, Laboratory for High Energy Physics, Bern, Switzerland \\ ${ }^{8}$ Department of Physics and Astronomy, University of Kentucky, Lexington, USA \\ ${ }^{9}$ Université Grenoble Alpes, CNRS, Grenoble INP, LPSC-IN2P3, Grenoble, France \\ ${ }^{10}$ Physics Department, University of Fribourg, Fribourg, Switzerland \\ ${ }^{11}$ H. Niewodniczanski Institute of Nuclear Physics, Polish Academy of Sciences, Kraków, Poland \\ 12 Department of Chemistry, TRIGA site, Johannes Gutenberg University Mainz, Mainz, Germany \\ ${ }^{13}$ Institut Laue-Langevin, Grenoble, France \\ ${ }^{14}$ Physikalisch Technische Bundesanstalt, Berlin, Germany \\ 15 Present address: Institute of Physics Belgrade, Belgrade, Serbia \\ ${ }^{16}$ Present address: CERN, Geneva, Switzerland
}

Published online: 21 July 2021

(C) The Author(s) 2021

\section{Erratum to:}

Eur. Phys. J. A (2021) 57:152

https://doi.org/10.1140/epja/s10050-021-00456-1

Unfortunately, the article contained an error in the affiliations of authors Z. Grujić and A. Schnabel.

In figure 3 the style of two vertical lines was changed from solid to dashed.

This has been corrected now and no other changes to the article have been made. We apologise for any inconvenience caused to our readers.

\begin{abstract}
Open Access This article is licensed under a Creative Commons Attribution 4.0 International License, which permits use, sharing, adaptation, distribution and reproduction in any medium or format, as long as you give appropriate credit to the original author(s) and the source, provide a link to the Creative Commons licence, and indicate if changes were made. The images or other third party material in this article are included in the article's Creative Commons licence, unless indicated otherwise in a credit line to the material. If material is not included in the article's Creative Commons licence and your intended use is not permitted by statutory regulation or exceeds the permitted use, you will need to obtain permission directly from the copyright holder. To view a copy of this licence, visit http://creativecomm ons.org/licenses/by/4.0/.
\end{abstract}

The original article can be found online at https://doi.org/10.1140/ epja/s10050-021-00456-1.

\footnotetext{
a e-mail: Jochen.Krempel@ phys.ethz.ch (corresponding author)
} 